\title{
Frontières
}

\section{La contemplation du cadavre dans la méditation bouddhiste et les arts visuels}

\section{Karine Delage}

Volume 23, numéro 1, automne 2010

Enquêtes sur le cadavre : 1 . Fascination

URI : https://id.erudit.org/iderudit/1004019ar

DOI : https://doi.org/10.7202/1004019ar

Aller au sommaire du numéro

\section{Éditeur(s)}

Université du Québec à Montréal

ISSN

1916-0976 (numérique)

Découvrir la revue

Citer cet article

Delage, K. (2010). La contemplation du cadavre dans la méditation bouddhiste et les arts visuels. Frontières, 23(1), 26-32. https://doi.org/10.7202/1004019ar
Résumé de l'article

Le cadavre possède un pouvoir d'évocation exceptionnel sur lequel s'appuient deux pratiques qui semblent éloignées à bien des égards : la contemplation de charnier, qui utilise les restes humains comme support visuel à la méditation sur la mort, et les productions contemporaines en arts visuels, qui manipulent ces restes comme matériau artistique. Ces deux pratiques remettent en question la frontière entre réfléchir sur la mort et réfléchir avec la mort, en développant des stratégies qui visent un double objectif : saisir l'essence de la mort et ainsi conjurer l'angoisse qu'elle génère. Dans cet article, notre objectif est de mettre en lumière des indicateurs révélant la place qu'occupent dans ces stratégies les trois gestes de la réduction phénoménologique selon Husserl : la conversion réflexive, la variation imaginaire et l'épochè transcendantale. 


\section{Résumé}

Le cadavre possède un pouvoir d'évocation exceptionnel sur lequel s'appuient deux pratiques qui semblent éloignées à bien des égards: la contemplation de charnier, qui utilise les restes humains comme support visuel à la méditation sur la mort, et les productions contemporaines en arts visuels, qui manipulent ces restes comme matériau artistique. Ces deux pratiques remettent en question la frontière entre réfléchir sur la mort et réfléchir avec la mort, en développant des stratégies qui visent un double objectif: saisir l'essence de la mort et ainsi conjurer l'angoisse qu'elle génère. Dans cet article, notre objectif est de mettre en lumière des indicateurs révélant la place qu'occupent dans ces stratégies les trois gestes de la réduction phénoménologique selon Husserl: la conversion réflexive, la variation imaginaire et l'épochè transcendantale.

Mots clés: cadavre - contemplation de charnier - arts visuels contemporains réduction phénoménologique imaginaire - dépassement de la limite.

\begin{abstract}
The corpse possesses an exceptional power of evocation on which are based two practices that seem to be very much far from each other: contemplation of charnel, that uses human remains as visual support for meditation on death, and contemporary productions in visual arts that use human remains as artistic materials. These two practices question the line between thinking with death and thinking of death by developing strategies that have a double objective: to grasp the essence of death and the anguish that it generates. In this article, our objective is to shed light on the indicators that reveal the occupied places of the three moments of husserlian phenomenological reduction in these strategies: reflexive conversion, imaginary variation and transcendental épochè.
\end{abstract}

Keywords: corpse - contemplation of charnel - contemporary visual arts - phenomenological reduction imaginary - exceeding limits.

\section{La contemplation du cadavre dans la méditation bouddhiste et les arts visuels}

\section{Karine Delage,}

étudiante à la maîtrise en sciences des religions, UQAM.

La méditation de charnier dans la tradition bouddhiste et les productions contemporaines en arts visuels qui usent de restes humains - réels ou symboliques - comme matériau artistique sont des manifestations qui demeurent éloignées à bien des égards, mais qui constituent toutes deux une recherche de sens à travers l'exploitation du cadavre. Le moine et l'artiste partagent des spécificités qui invitent à penser leur rapprochement ${ }^{1}$. Toutefois, dans cet article, nous nous intéressons moins aux personnages qu'au déploiement d'une pratique qui s'élabore à partir de restes humains et qui est engagée dans une réflexion sur la mort. Ces méthodes de méditation et ces productions contemporaines en arts visuels témoignent d'un déploiement imaginatif exceptionnel pour percer l'essence de la mort. On peut se demander jusqu'à quel point ces pratiques de constitution du sens font appel aux trois formes de la réduction phénoménologique selon la méthode husserlienne : la conversion réflexive, la variation imaginaire et l'épochè transcendantale. Un examen de ces pratiques de méditation et de quelquesunes de ces œuvres d'art permet de repérer les indicateurs d'une réduction phénoménologique et de constater comment chacun des trois gestes de modification de la conscience que permet la méthode phénoménologique contribue à un déploiement méthodique de l'imaginaire sur le cadavre.

\section{PRÉSENTATION DES PRATIQUES}

\section{LE CADAVRE DANS LA MÉDITATION BOUDDHISTE THERAVADA}

Selon le bouddhisme theravada, la mort est au cœur des quatre révélations qui auraient déclenché la quête spirituelle du Bouddha. La maladie, la vieillesse, la mort et l'ascétisme sont condensés dans l'idée fondatrice de cette doctrine: la conscience de l'éphémère. Ainsi, pour les dévots laïcs et les moines, la méditation prenant la mort comme sujet est inséparable de cette pratique et elle prend parfois la forme plus 


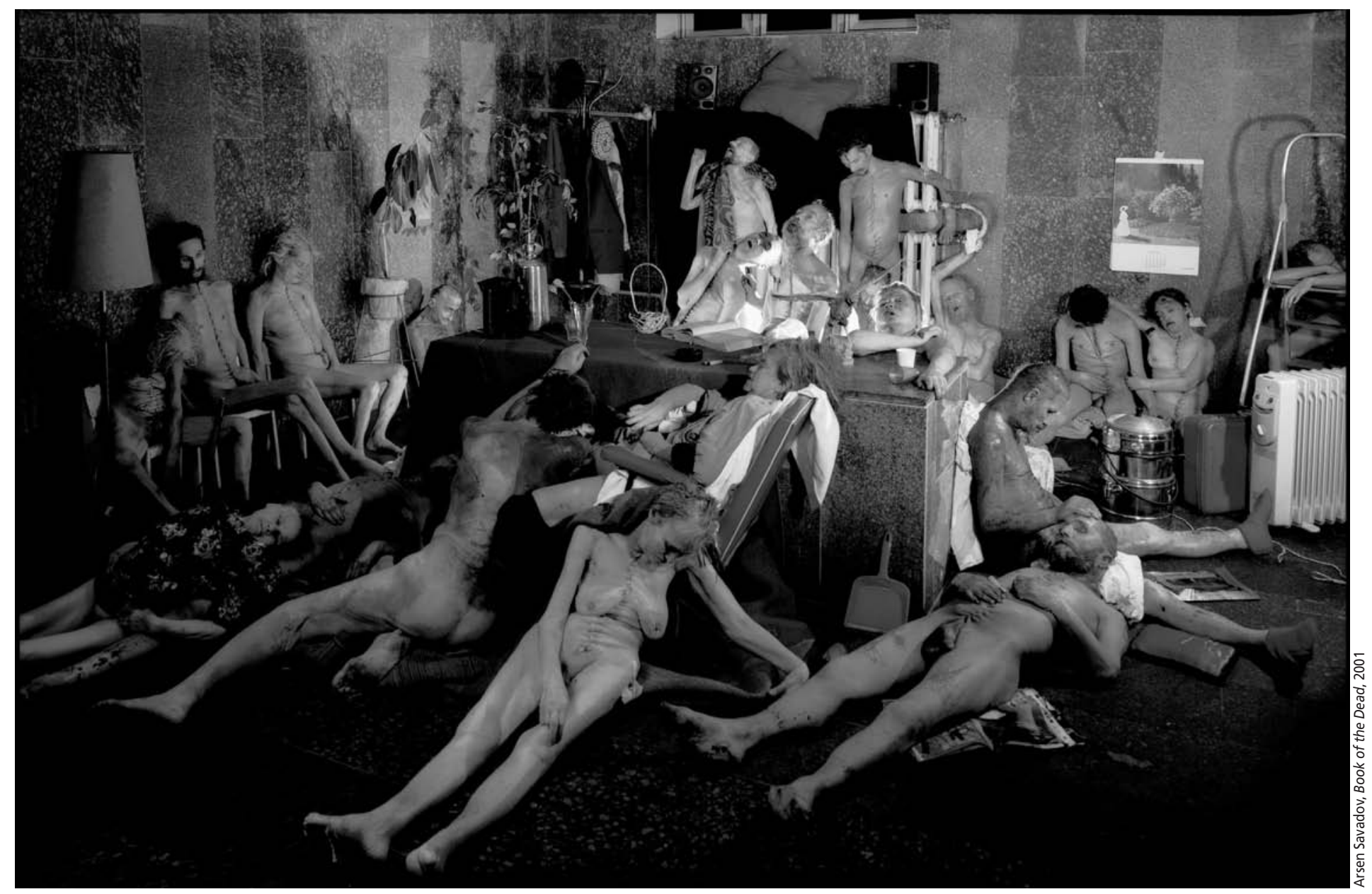

extrême de la méditation de charnier ${ }^{2}$. Le cadavre y joue un rôle essentiel en devenant le support visuel d'une réflexion sur l'évanescence du corps et en agissant comme un adjuvant de la conscience de l'éphémère.

Des écarts normatifs importants sont à noter entre les écrits canoniques et postcanoniques palis ${ }^{3}$. Le passage canonique sur les neuf contemplations de charnier «ne prescrit pas explicitement la contemplation directe de cadavres» alors que dans le texte postcanonique l'«observation de cadavres devient un aspect important de cette contemplation» (Boisvert, 1995, p. 33). Les enjeux relatifs à une telle pratique sont pour leur part sans équivoques : la méditation de charnier permet de « développer le dégoût envers notre propre corps et celui des autres afin d'éteindre tout désir ou passion» (Boisvert, 1995, p. 33). Cette conscience de l'asubha peut mener au nirvana. Des témoignages confirment l'importance que garde encore aujourd'hui la méditation de charnier (Klima, 2002; Boisvert, 1995).

\section{LE CADAVRE DANS LES ARTS VISUELS CONTEMPORAINS}

Les œuvres de certains artistes contemporains s'inscrivent à l'intérieur d'une démarche où apparaît cette même tension entre la réflexion sur la mort et la proximité du cadavre. La naissance de l'art, comme le rappelle l'historien de l'art JeanPierre Latour, est intrinsèquement liée à la réflexion sur la mort. Bien que l'art ait peu à peu délaissé sa fonction rituelle au profit d'un art séculier, plusieurs productions contemporaines en arts visuels continuent de s'inscrire dans une pratique de symbolisation de la mort et de recherche de sens devant le vide qu'elle laisse. Dans le monde de la représentation, ce vide touche également l'iconicité, c'est-à-dire l'irreprésentabilité de la mort en soi, qui constitue un enjeu fondamental de la problématique entre art et mort.

Les artistes qui travaillent cette problématique s'intéressent, à travers leurs œuvres, au passage de l'état vivant à l'état mort. Ce faisant, ils explorent les limites de la promiscuité avec la mort notamment à travers le cadavre - réel ou symbolique. Ainsi, un grand nombre d'œuvres performatives sont créées par la voie des expériences à risque, où l'intégrité physique de l'artiste est compromise. Parmi ces productions $^{4}$ : Solo pour la mort (1964) où Serge III Oldenbourg performe un tir de roulette russe ${ }^{5}$, Shout (1971) où Chris Burden est blessé par balles, ou encore l'ascèse périlleuse de David Nebreda ${ }^{6}$.
Une autre voie témoignant de ce rapport direct avec la mort est celle de la manipulation des restes humains. Le Livre des Morts (2001) où Arsen Savadov met en scène des cadavres grossièrement recousus dans des poses naturalistes ${ }^{7}$ et la Table à deux bancs (2005) de Teresa Margolles, qui récupère pour ses œuvres les fluides organiques d'autopsies pratiquées dans le cadre de son travail de médecin légiste, en sont des exemples. Ainsi, en dépit des moyens empruntés, les artistes ont bien conscience que traquer la mort constitue un outil rhétorique extrêmement puissant.

La mort, et plus spécialement les restes humains, forment effectivement un support sensible sur les plans symbolique, social, politique et éthique que les méthodes méditatives et les productions artistiques mettent à profit de manière différente. Cela dit, dans chacun de ces cas, il semble qu'une frontière soit franchie entre travailler sur le thème de la mort et travailler avec la mort.

\section{DES CONVERGENCES}

Pour la pratique méditative comme pour la pratique artistique, la fréquentation du macabre est intrinsèquement liée à l'angoisse générée par la mort.

La méditation de charnier permet de prendre conscience de l'évanescence de 
la vie. Cette démarche vise à cultiver une forme très accomplie de détachement (Klima, 2002, p. 187), apaisant ainsi l'angoisse de se savoir voué à la mort. L'anthropologie reconnaît aussi la fonction conjuratoire de l'art, que ce soit en nommant, "au-delà du corps éteint, une transcendance et une réponse au mystère de l'existence humaine » (Des Aulniers, 1996, p. 12), en donnant «un sens à la mort. Quel que soit ce sens!» (Des Aulniers, 1996, p. 11) ou en apprivoisant «la mort comme un phénomène douloureux mais appartenant à la vie » (Des Aulniers, 1996, p. 14). Les efforts mis en œuvre dans la pratique méditative et dans les productions artistiques pour répondre à l'angoisse de la mort convergent: ces deux types de démarches multiplient et intensifient les rapprochements avec le macabre pour saisir la nature et la signification de la mort.

\section{DES DIVERGENCES}

Les pratiques méditatives et artistiques divergent par ailleurs sur deux points. Premièrement, la notion de cadavre se situe dans des cadres de référence différents. Dans la pensée bouddhiste, tout ce qui vit est à la fois souffrance, éphémère et dénudé de soi. Dans une telle perspective l'individualité perd toute valeur; la notion de «personne» renvoie plutôt à un agrégat psycho-physique de matière, de sensations, d'activités karmiques et de niveaux de conscience, qui naît et meurt continuellement. Le cadavre semble donc agir comme l'ultime illustration de la doctrine bouddhiste: il exacerbe l'évanescence à travers la putréfaction, il ne souffre plus et ne possède aucune individualité persistante - il ne saurait être cette figure d'une existence passée ${ }^{8}$

La notion de cadavre que véhiculent les propositions en art contemporain est bien différente. Le cadavre est cette «figure réifiée de l'être qui ne pense plus mais toutefois existe, qui abdique toute spiritualité mais reste matière [... il] est l'énigme de ce qui à la fois est et n'est pas » (Ardenne, 2006, p. 394). Dans ce cadre de référence, on insiste sur la problématique de la disparition du mort et de son histoire. L'empathie à l'égard de notre semblable persiste par-delà sa mort, traduisant en fait nos préoccupations à l'égard de notre propre personne, de notre individualité singulière qui risque aussi de disparaître.

Ces cadres de références sont aussi ouverts à des métissages. Le trio d'artistes chinois Cadavre est en ce sens exemplaire. Leur compréhension du cadavre fusionne une forme de détachement proche de la pensée bouddhiste et un type de regard biologisant proche de la médecine occidentale. Quatre postulats sont alors avan- cés pour justifier l'utilisation de restes humains comme matériau artistique : 1) le corps matériel est le support de l'homme spirituel; 2) ce dernier disparaît avec la mort du premier; 3) sur le plan conceptuel, le cadavre n'est plus un humain, mais sur le plan psychologique, pour le vivant, il le demeure, d'où la spécificité et l'intérêt de ce support; 4) c'est dans la fonction de l'art de transgresser les tabous (Dawei, 2001, p. 61).

Le deuxième élément de divergence entre les pratiques méditatives et artistiques concerne les solutions mises de l'avant pour répondre à l'angoisse de la mort qu'elles partagent. Dans la méditation, l'objet funèbre doit permettre au pratiquant de se détacher de tout désir ou affection pour ce qui est éphémère, notamment son individualité existentielle. Dans l'art, les restes humains ou animaux opèrent plutôt comme contre-exemple afin d'exalter la vie et comme monument pour la pérennité. L'artiste Teresa Margollez décrit d'ailleurs son travail sur les cadavres comme une tentative de prolonger la vie (Cuir, 2001, p. 70). Deux registres différents de valorisation - la mort et l'éphémère; la vie et la pérennité - sont donc proposés pour répondre à une même angoisse.

Toutefois, malgré les stratégies mises en place, la difficulté d'accepter la mort transparaît quand les pratiques aboutissent à une momification, littérale dans le cas de certains moines (Georges, 1982, p. 251-254), symbolique dans le cas de certaines œuvres d'art. Le travail de méditation sur l'éphémère semble ici courtcircuité. Le corps du moine demeure, figé dans son processus de disparition. Qui plus est, un culte - marquant une idéalisation, un attachement, une affection - est aussi voué à ces moines et moniales momifiés, confirmant ainsi l'exemplarité de leur cheminement ${ }^{9}$.

Cette négation de la mort est également présente dans l'art. Pour Ardenne, les «représentations artistiques du cadavre humain, y compris les plus morbides et les plus antihumanistes qui soient [...], expriment une évidente difficulté à voir mourir»(Ardenne, 2006, p. 410). Ainsi, la «représentation du cadavre [...] joue un rôle proche de la momification [...] et de maints autres rites funéraires de conservation» (Ardenne, 2006, p. 409). De sorte que le cadavre artistique, paradoxalement, résiste au temps et immortalise le périssable.

Les pratiques méditatives et artistiques, tant dans les caractéristiques qu'elles partagent que dans celles qui les distinguent, mettent à profit toutes les ressources de l'imagination pour percer le mystère de la mort, pour accéder à son essence, pour produire du sens à partir du «hors sens » qu'est la mort. C'est à cet égard que la phénoménologie husserlienne, axée sur la constitution du sens à partir de l'expérience vécue, peut être mise à contribution. La méthode qu'elle propose, la réduction phénoménologique, se veut selon Depraz «une pragmatique à part entière » (2001, p. 25), une «pratique concrète» (2001, p. 25) qui pourra fournir un éclairage utile sur les pratiques qui nous intéressent ici: la méditation de charnier et la production artistique usant de restes humains.

\section{RETRACER LES TROIS GESTES DE LA RÉDUCTION PHÉNOMÉNOLOGIQUE DANS LA MÉDITATION DE CHARNIER ET DANS LA PRODUCTION D'CEUVRES D'ART À PARTIR DE RESTES HUMAINS}

La phénoménologie husserlienne étudie la constitution du sens à partir de l'expérience vécue et propose une méthode, la réduction phénoménologique, qui se compose de trois gestes intérieurs ou mentaux: la conversion réflexive, la variation imaginaire et l'épochè transcendantale. Depraz insiste sur le fait que cette démarche de pensée est aussi une pratique bien concrète:

Une des tâches possibles pour le phénoménologue d'aujourd'hui consiste donc à faire ressortir la dimension de pratique concrète à l'œuvre implicitement dans cette démarche de pensée, révélant ce faisant la phénoménologie comme une pragmatique à part entière. [...] la réduction peut être pratiquée consciemment comme un exercice quotidien et [...] c'est à cette seule condition que la phénoménologie est véritablement à la hauteur de son exigence fondatrice (2001, p. 25).

On peut se demander si les témoignages des méditants ainsi que les œuvres des artistes peuvent être considérés comme une description des vécus subjectifs comparable à ce que la réduction phénoménologique permet d'obtenir. Ces témoignages et ces œuvres rendent-ils compte d'un processus intérieur et mental qui se rapproche des trois gestes de la réduction phénoménologique? Des exemples tirés de la contemplation de charnier et de l'art contemporain nous permettront d'examiner comment chacun des trois moments de la réduction phénoménologique est mis à contribution.

\section{LA CONVERSION RÉFLEXIVE:}

\section{SE RETOURNER VERS SOI}

Le premier geste de la réduction phénoménologique «marque une disconti- 
nuité avec le régime perceptif des objets » (Depraz, 1999, p. 25). La conversion réflexive modifie l'objet de notre attention ; le mouvement de la pensée ne va plus vers le perçu, mais vers l'expérience subjective engendrée par l'objet ou par la situation observé. Elle «permet de [se] désengager tant soit peu de la fascination qu'exerce le monde et ses objets, pour [...] prendre en vue les actes et les vécus eux-mêmes» (Depraz, 1999, p. 26).

Il est instructif de considérer la fonction répulsive du cadavre dans la contemplation de charnier et dans les productions en arts visuels à la lumière de cette attention portée au vécu. Comme le "Convertere renvoie à ce geste de se tourner vers soi en se détournant du monde» (Depraz, 1999, p. 26), le «non-beau » et le «non-présentable» agissent ici comme repoussoir. Ce faisant, méditants, artistes et spectateurs sont alors invités à se "détourner» du monde pour s'intéresser à ce qui se passe en eux pendant qu'ils observent un cadavre.

Le témoignage d'un moine méditant, recueilli par Boisvert (1995), va dans le sens d'une telle démarche. À la suite d'une observation de dissection en salle d'autopsie, le moine souligne l'importance

[...] d'identifier les émotions, sentiments et images qui nous viennent à l'esprit. Pour transformer cette expérience en méditation, d'après le moine, il faut être constamment attentif à ses émotions. Ceci permet de développer une compréhension de la réalité telle qu'elle est sans toutefois engendrer de haine ou de dégoût (Boisvert, 1995, p. 35).

Le témoignage du moine Luang $\mathrm{Bu}$ Sangwaan rend également compte d'un mouvement de «conversion», qui part de ce qui est perçu pour faire retour sur l'acte intérieur qui est accompli (rapporté par Klima, 2002, p. 176-181). Ce dernier dit avoir fait le choix de passer six années en retraite dans un charnel ground ${ }^{10}$ afin de se confronter à l'angoisse et à l'horreur que suscite un tel environnement. Son expérience a été marquée par une attention portée à chaque parcelle de vécu pouvant perturber ses objectifs de méditation afin d'en saisir la source et ainsi s'en libérer.

Dans la production artistique, le geste posé pour produire une représentation se rapproche de la conversion réflexive, qui «décrit la conscience en train d'opérer un tour quasi complet sur elle-même; quasi, parce qu'elle ne s'y enferme pas à nouveau, mais trace ce cercle depuis la visée du monde, et y retourne toujours en dernière instance»(Depraz, 1999, p. 26). Le fait même de re-présenter fait écho au mouvement de conversion réflexive, il est cette distanciation prise à l'égard de l'ob- jet matériel. Par ailleurs, l'œuvre en étant rendue disponible au regard du spectateur, réintroduit le monde, qui peut à nouveau donner lieu à une autre conversion réflexive.

Enfin, la réitération propre aux pratiques méditatives et artistiques rend compte de la fragilité spécifique de la conversion réflexive. Cette difficulté à maintenir l'attention dans le vécu est due au caractère contingent du vécu observé et à la difficulté de maintenir dans le temps une telle observation. L'introduction du second geste de la réduction phénoménologique vient toutefois consolider le retournement vers soi.

\section{LA VARIATION IMAGINAIRE: \\ LE DÉFILEMENT MENTAL DU POSSIBLE}

Pour révéler des vécus subjectifs dans leurs caractéristiques universelles et essentielles, l'imagination devient ici un outil indispensable. La variation imaginaire, ou variation eidétique, vient palier la contingence d'une situation et la concrétude de l'objet en faisant défiler mentalement toutes les expériences passées ou possibles pour un type de situation. Les exemples qui suivent permettent d'illustrer le travail immense de l'esprit pour répondre à la fascination qu'exerce la mort sur l'esprit et sur les sens.

La littérature sutta (discours du Bouddha) indique comment la réflexion sur le mode de la variation imaginaire peut être mise en œuvre selon le canon pali. Neuf variantes de la contemplation de charnier y sont présentées sous la forme d'une classification en neuf degrés de décomposition. Ceux-ci doivent aider le méditant à se projeter dans chacun de ces moments afin d'éprouver et accepter sa propre évanescence. Le texte stipule que « [s]i un moine voit un cadavre abandonné dans un charnier [...], il contemplera son propre corps en pensant: " ce corps est de même nature que celui que je vois et un jour lui deviendra semblable, c'est inévitable"»(Boisvert, 1995, p. 33). Cette méthode est codifiée et systématisée dans l'exégèse du canon pali. D'une part, «dix différents stades de décomposition sont classifiés [...]: les corps boursouflés, livides, ulcérés, déchiquetés, rongés, éparpillés, mutilés et éparpillés, saignants, infestés de vers et les squelettes » (Boisvert, 1995, p. 33). La contemplation de charnier évolue selon des étapes précises et non pas au gré du hasard:

[...] si la méditation du yogi n'est pas couronnée de succès, il doit alors entrevoir la mort sous différentes approches : 1) comme étant un meurtrier (qui apparaît soudainement et enlève la vie), 2) comme étant la ruine du succès (car la mort détruit tous les accomplissements), 3) par comparaison (c.-à-d. en se comparant à ceux qui sont déjà morts), 4) comme étant un partage du corps avec plusieurs [...], 5) en établissant un parallèle avec la fragilité de la vie, 6) comme étant imprévisible, 7) comme reflétant la limitation de la vie et 8 ) comme étant l'évanescence de chaque moment, car un individu n'est vivant que pour la durée d'un moment (Boisvert, 1995, p. 34).

Le déploiement de l'imaginaire est également à l'œuvre dans l'aménagement de l'espace de méditation. L'étude de Klima (2002) montre combien l'environnement du méditant est un élément central pour l'éveil de la conscience de l'éphémère. L'anthropologue écrit: «La première raison de fréquenter un endroit où l'on dispose de cadavres ou d'habiter dans un temple chargé d'objets évocateurs de la mort est qu'ils facilitent délibérément la construction des images nimitta ${ }^{11}$ » (Klima, 2002, p. 181; notre traduction).

Dans les temples dédiés à la méditation sur la mort, on trouve des mises en scène d'images mortuaires, d'ossements et de momies. Lors des recherches de Klima au Toong Sammakhi Dhamm, le temple mettait à la disposition des méditants :

un cadavre avec la chair ouverte, révélant les entrailles; un autre placé sur une table avec des lambeaux de viande calcinés pendant des os; un couché sur son ventre avec de la chair grise bouffie et gonflé; des photos encadrées de cadavres alignées sur l'autel du petit hall de méditation; une paire de squelettes humains complets, suspendus du plafond [...] (Klima, 2002, p. 172 ; notre traduction).

Cette cumulation d'objets facilite le déploiement d'une variation en imagination sur la mort. Les enseignements des maîtres yogis révèlent par ailleurs une richesse imaginative qui tend vers une autonomie par rapport à de tels supports matériels. "Dans asubha Kammatthana, on reçoit la consigne de visualiser en alternance cinq parties extérieures du corps: cheveux, poil de corps, ongles, dents et peau» (Klima, 2002, p. 181; notre traduction).

Selon ces enseignements, la richesse évocatrice de ces éléments tient, d'une part, à la familiarité visuellement de ces derniers et, d'autre part, à la matière morte qui les compose. Ainsi, comme l'écrit Depraz, «c'est le pouvoir indéfini de l'imaginaire qui permet de saisir l'essence [...], car il ne s'agit pas d'aller observer concrètement tous les [cadavres, dans une infinité de 
postures improbables] pour ressaisir leur invariant» (Depraz, 1999, p. 58).

Pourtant, dans les pratiques contemporaines, le contact avec le cadavre tend à s'extrémiser, et l'exercice de l'imagination ne semble plus servir de «garde-fou contre l'empirisme ${ }^{12}$. L'exemple suivant nous semble confirmer cette réalité. Effectivement, si léguer sa dépouille à sa communauté monastique afin de lui faire don d'un support pour la méditation du «non-beau» paraît un geste bienveillant, les évènements que Klima rapporte après la mort d'une moniale sont tout à fait inusités. Il écrit:

D'abord, les moines et les moniales placèrent le corps à l'extérieur, sur une table de bambou, pour le regarder se décomposer durant un moment. Par la suite, ils bouillirent le cadavre dans une grande marmite. Ils sortirent le cadavre cuit avec des morceaux de chairs calcinés pendants, et le remirent sur la table pour l'observer à nouveau. Ils prirent un fendoir à viande pour écorcher la peau brûlée, le gras grisonnant et les muscles de ses os. Ils arrachèrent à mains nues tous les intestins et les organes internes du cadavre. Finalement, ils bouillirent et nettoyèrent les os, les attachèrent ensemble, et les accrochèrent dans le hall de méditation (Klima, 2002, p. 205-206; notre traduction).

Ces différentes manipulations sont photographiées afin de servir ultérieurement comme supports visuels à la méditation. Ce type de mise en scène poussée à l'extrême se retrouve également dans la pratique artistique.

Dans la fabrication de l'improbable, certaines productions artistiques sont manifestes. La performance de l'artiste, l'installation, la photographie, la vidéo, les arts graphiques, mais aussi toutes les propositions théoriques qui en découlent, constituent autant de supports pour rendre compte de la moindre variation imaginaire sur la mort et où le cadavre est souvent la vedette.

La cartographie des représentations du cadavre proposée par Ardenne illustre bien le travail de l'esprit que nous décrivons ici. L'anthropologue départage les représentations idéalistes (la belle-mort, le beau-mort, le beau-cadavre) des représentations matérielles (le corps-matière, le corps-viande, le corps-fluide), et il place entre les deux les représentations du «passage » où le corps passe du beau au putride, de l'extérieur à l'intérieur (Ardenne, 2006, p. 397). «[T]racée par la loi de la dégénérescence, implicite au destin du cadavre» (Ardenne, 2006, p. 397), cette classifica- tion rappelle celles présentées dans la littérature canonique et postcanonique palie.

Nos recherches sur des œuvres d'artistes contemporains permettent également d'observer le déploiement de l'imagination selon une typologie qui retient six formes de représentation de la mort dans les productions artistiques. Les cas de figure retenus à cet effet tendent de plus à confirmer l'idée selon laquelle les représentations métaphoriques de la mort présentent une plus grande efficacité évocatrice pour en libérer l'essence (Des Aulniers et al., 1996, p. 14). Effectivement, la taxidermie, les produits organiques animaux et humains ainsi que le cadavre en soi témoignent d'une forte valeur évocatrice et réflexive sur la mort, alors que la nécrophilie et l'anthropophagie - deux pathologies avérées - produisent une aversion telle qu'elles n'induisent plus une réflexion sur la mort en soi. Dans ces deux cas particuliers, la méthode husserlienne prend tout son sens dans la mesure où elle doit permettre l'économie d'expérimentations empiriques excessives. Depraz souligne d'ailleurs que la «variation est imaginaire: elle révèle que les possibilités libérées par l'imagination dépassent de beaucoup ce que l'empirie permet»(Depraz, 1999, p. 58; notre soulignement).

Ainsi, comme dans le cas de la dépouille de la moniale, quand l'artiste chinois Zhu Yu cuisine et mange un fœtus, il n'est pas certain qu'une quête de l'eidétique de la mort soit en jeu. Selon Depraz, une variation dite eidétique est une opération mentale "par laquelle je cesse de voir dans chaque objet ou situation un fait particulier pour l'envisager comme une structure essentielle et universelle de mon expérience»; les trois gestes mentaux de la réduction phénoménologique mènent à une procédure discursive bien précise: la description du phénomène en cause (Depraz, 2001, p. 25). L'inclusion de certaines pratiques peut donc poser problème, et nous pouvons nous demander si l'actualisation de l'imaginaire va trop loin dans la recherche de l'essence. De surcroît, de quelle essence est-il question? Par exemple, les propos derrière l'œuvre de Yu donnent à cette mise en scène du cadavre un effet repoussoir bien différent de celui que produit la méditation bouddhiste, puisque l'artiste cherche délibérément la provocation par la transgression du tabou de l'anthropophagie. Ainsi, le dégoût provoqué n'est plus contre l'évanescence de la vie, mais il vient célébrer la valeur de l'être humain et de ses tabous ${ }^{13}$.

Les œuvres à caractère nécrophile soulèvent le même type de questions. Par exemple, dans Blind Date (1980), John Ducan place dans son itinéraire vers l'infertilité un coït nécrophile avant sa vasectomie ${ }^{14}$. Bien qu'articulée dans une démarche artistique orientée sur la question de la procréation, cette œuvre emprunte la forme de la déviance pour inscrire son message. Autre exemple, Le Livre des Morts (2004) de Savadov, cité plus tôt, présente une allégorie contemporaine de la danse macabre, mais celle-ci se fait néanmoins par une esthétisation du cadavre proche des pratiques de nécrophiles notoires ${ }^{15}$.

En tant que "procédures discursives", ces échappées artistiques ne semblent pas correspondre à une variation eidétique sur le phénomène de la mort. Il semble que l'on soit en présence de stratégies qui cherchent davantage à affirmer la vie, l'horreur, le tabou, la limite dépassée, etc.

Quelques cas de figure évocateurs sont à chercher dans les quatre éléments typologiques restants. Par exemple, le cadavre animal est un matériau récurrent chez certains artistes. L'usage de la taxidermie se retrouve notamment dans la série pensée par Damien Hirst dans laquelle des mammifères et amphibiens (entiers ou tranchés) sont conservés dans le formol et exposés à travers des boîtes hermétiques vitrées ${ }^{16}$; dans les sculptures de Jan Fabre créées à partir d'écailles de bousier et d'animaux empaillés; ou encore dans le suicide anthropomorphisé d'un écureuil de Maurizio Cattelan (Bidibidobidiboo, 1996). Ici, la mort animale crée une distance confortable, toutefois la question de la mort humaine surgit subrepticement pour confronter le spectateur, par exemple, à travers le titre, les figures funéraires humaines (crâne humain, cercueil) ou encore dans la mise en scène domestique d'un suicide.

Les produits organiques animaux et humains sont aussi des matériaux importants dans l'art contemporain. Par exemple, Jana Sterback ${ }^{17}$ performait en 1987, Vanitas - Robe de chair pour albinos anorexique (1987). L'artiste féministe avait alors revêtu un vêtement de steaks cousus qu'elle avait confectionné. Robert Gligorov $^{18}$, dix ans plus tard, attend, vêtu de son Jacket-waiting (1997), que la vermine dévore son manteau. Gligorov met alors davantage l'accent sur le passage de la viande saine à la viande putride. Par ailleurs, la matière organique prend une tout autre charge symbolique quand elle est isolée du corps humain qui la maintenait en vie. C'est cet effet étrange peutêtre que réussit à produire Self (1991), une œuvre dans laquelle Damien Hirst modèle et sculpte sa tête à partir du placenta de son premier enfant.

Enfin, la représentation spécifique du cadavre constitue l'espace où se joue 
la plus grande tension symbolique et, conséquemment, l'espace d'où vraisemblablement naissent des problématiques semblables à celles posées par les œuvres de Yu, Ducan et Savadov. La mise en place d'une distance dans la représentation du cadavre par la métaphore, l'esthétisation, la ritualisation, le médium, semble toujours rendre les œuvres plus supportables et, dès lors, plus évocatrices. À ce titre, il faut compter Dead Dad (1996-1997) où Ron Mueck représente le cadavre de son père. Dans cette œuvre, l'hyperréalisme du mannequin est trahi par la miniaturisation de la forme humaine - le père mesure à peine un mètre. Le corps encore lisse et beau participe à cette distance avec l'intenable. Les séries La morgue d'Andres Serano et La vie avant la mort de Walter Schels sont des œuvres exemplaires d'esthétisation du cadavre à travers le médium de la photographie. L'idée de la mort prend également forme dans le thème de l'artiste en cadavre, comme dans Untitled 1531 (1981), où Cindy Sherman ${ }^{19}$ s'autoportraiture en proto-cadavre abandonné sur un sol terreux visiblement victime d'un viol meurtrier.

Ces quelques exemples empruntent des stratégies différentes pour traduire et induire une réflexion sur la mort. Par ailleurs, toutes ces œuvres suggèrent que la méditation sur la mort ne fonctionne qu'à la condition d'y mettre un peu du sien: s'identifier à une figure macabre, porter la mort en revêtant de la chair animale, modeler les restes organiques de ses proches ou encore acter son propre cadavre. Dans le vocabulaire de la réduction phénoménologique, nous pourrions dire que cet «acte imaginatif se présente en fait comme un cas délimité d'épochè, une sorte de propédeutique partielle à l'accomplissement radical, définitif et universel de l'épochè transcendantale» (Depraz, 1999, p. 58).

\section{L'ÉPOCHĖ TRANSCENDANTALE \\ L'ABOLITION DU MONDE ET DE SOI}

Pour Depraz, l'épochè transcendantale est le geste à la fois inaugural, terminal et total de la méthode phénoménologique. Ce geste consiste en une mise hors circuit de " tout ce que nous pensons spontanément comme une validité à nos yeux » (Depraz, 1999 , p. 28). Volontaire ou imposée par des évènements extérieurs, cette interruption des pensées habituelles et des actions quotidiennes fait de l'épochè le geste réductif de base. Cette suspension est «une activité continue et définitive» (Depraz, 1999, p. 29), qui participe à tout acte de remémoration ou d'imagination. L'épochè transcendantale traverse donc la conversion réflexive et la variation eidé- tique. Sa radicalité tient à la portée de la neutralisation qui atteint la validité des objets du monde, le monde en soi et l'ego même de l'acteur opérant. Depraz écrit:

La réduction est transcendantale à

la mesure du «résidu» qu'elle laisse indemne de cette destruction radicale: la conscience égologique pure. [...] Je me mets moi-même en suspens en tant qu'ego naturel absorbé dans le monde, je crée la fiction de mon propre anéantissement, pour renaître indemne, en tant qu'ego transcendantal (Depraz, 1999, p. 29).

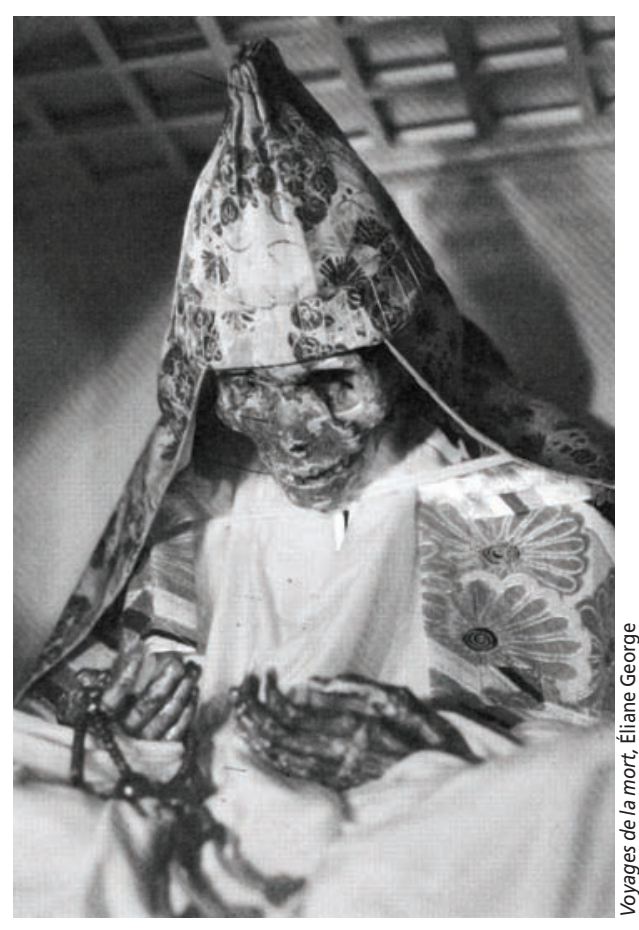

Ceci résonne de manière étonnante avec certains éléments des pratiques à l'étude qui mettent le rôle de l'imagination au profit d'une expérience empathique. Depraz précise d'ailleurs que la fonction méthodique de l'imagination permet une distorsion à l'extrême «dans [son] unité égoïque pour aller en fiction au lieu occupé par autrui sans pour autant devenir autrui, sans détruire [son] unité » (Depraz, 1999, p. 59). Dans certaines méditations et certaines productions en arts visuels, cette fiction, ce lieu occupé par autrui, c'est la mort. À travers différents moyens, les méditants et les artistes tentent ainsi de se mettre (de nous mettre) dans la peau d'un mort, voire paradoxalement d'éprouver l'expérience d'être un cadavre. Enfin, parce qu'on ne peut être témoin de la mort, la réflexion même sur elle implique toujours et nécessairement une fiction, une projection de soi dans la mort même ou dans les deuils qu'elle provoque. Comme l'écrit Ardenne, à «moins de se supprimer soi-même, expérimenter le " mourir" com- mande tout à la fois la dérogation et le spectacle: il faut que quelqu'un meure et que je puisse le regarder mourir» (Ardenne, 2006, p. 363). Il faut qu'il y ait épochè transcendantale, c'est-à-dire anéantissement et reconstruction du monde (Depraz, 1999, p. 29) à travers les pratiques méditatives et les productions artistiques.

$$
* * *
$$

Marginales à l'intérieur même des champs de pratique auxquels elles appartiennent, la contemplation de charnier et les productions à partir de restes humains ou animaux en arts visuels entretiennent une relation de cohabitation avec les figures de la mort. Prétendant conjurer l'angoisse engendrée par notre condition de mortel en tentant de saisir l'essence de la mort, ces pratiques se sont approprié un objet d'une puissance évocatrice exceptionnelle: le cadavre. Dans ces deux types de pratiques, l'imagination travaille à varier les multiples possibilités de cette matière et ainsi générer une gamme extraordinaire d'expériences et de réflexions à l'égard de la mort. Le travail d'attention ainsi demandé au moine et à l'artiste - ainsi qu'au spectateur de l'œuvre - n'est-il pas utopique? La seule attitude qu'il conviendrait de garder devant la mort, n'est-elle pas la pensée de l'angoisse, comme le propose Gadamer (1995, p. 125)? Force est d'admettre que l'«extrémisation» de ces pratiques tend à montrer l'insuccès de leur ambition; aucune d'elles ne semble être parvenue à conjurer l'angoisse de la mort. Qui plus est, la culture de la transgression des tabous, propre au contexte de l'art contemporain, encourage également cette surenchère de la limite dépassée.

Notre examen de certaines pratiques par rapport à ce que vise la variation imaginaire a montré que la matérialité du cadavre ne constituait pas toujours une voie royale pour atteindre l'essence de la mort. L'éviscération artisanale et la cuisson de cadavres font basculer la contemplation de charnier dans la région obscure de la nécrophilie. Dans de telles ritualisations, les méditants occultent un déni du mourir; comme le nécrophile, il rend durable et perpétue «ce que la nature a voulu périssable [...], il continue à en jouir comme d'un sujet vif» (Ardenne, 2006, p. 419). Ainsi adossée aux squelettes suspendus dans les temples et aux grands maîtres bouddhistes momifiés pour être présentés à la vénération des fidèles, la prédication de la doctrine bouddhiste de l'éphémère devient paradoxale.

Dans l'art, les représentations du cadavre - qu'elles soient acceptables ou intenables - peuvent aussi être mises au profit d'une réflexion sur la vie: sa beauté, sa puissance et la sentimentalité qui s'y 
rattache, comme ses limites et ses tabous. Les cas de figure où les caractéristiques eidétiques de la mort semblent effectivement visées empruntent davantage les voies de la métaphore ou de l'esthétisation du cadavre que la crudité du propos.

Ainsi, peut-on conclure que lorsque «la mort devient un objet de vénération tel que la vie même semble la jalouser, et l'idolâtrer plus qu'elle-même, il y a en tout état de cause matière à méditer » (Ardenne, 2006, p. 431). Conviendrait-il de rappeler aux méditants et aux artistes que la variation imaginaire constitue, selon l'expression de Depraz, «un garde-fou contre l'empirisme» (1999, p. 26) et que l'essence ne se trouve pas dans l'observation empirique de chaque situation, mais bien dans le pouvoir de l'imagination que leurs pratiques respectives, justement, appellent?

\section{Bibliographie}

AKINCANO, T.A.A. (s.d.). "Glossary », en ligne, <http://www.meditationsheffield. com/Books5/Ajahn_Anand_Akincano_ Glossary.htm>, What-Buddha-Taught.net, consulté 2010-04.

ARDENNE, P. (2006). Extrême: esthétiques de la limites dépassée, Paris, Flammarion.

ARDENNE, (1999). "Expérimenter le réel. Art et réalité à la fin du $\mathrm{XX}^{\mathrm{e}}$ siècle », Pratiques contemporaines l'art comme expérience, Paris, Dis voir.

BOISVERT, M. (1995). "Bouddhisme, contemplation et mort », Frontières, vol. 7, no 3, p. 32-37.

CALLAGAN, L. (2010). «Six artists explore the sights and sounds of silence in Atmosphères ", The Gazette, 3 avril 2010, p. E10.

CUIR, R. (2001). "SEMEFO, Art after death: Traces de corps", dans Richard LEYDIER (dir.), Représenter l'horreur, Paris, Art Press, p. 65-70.

DAWEI, F. (2001). «Transgresser le principe céleste. Dialogue avec le groupe Cadavre», dans Richard LEYDIER (dir.), Représenter l'horreur, Paris, Art Press, p. 60-64.

DEPRAZ, N. (2001). "Une pratique concrète", Magazine littéraire: La phénoménologie, no 403, novembre, p. 25-26.

DEPRAZ, N. (1999). Husserl, Paris, Armand Colin, coll. "Synthèse, série philosophie ».

DES AULNIERS, L. et al. (1996). «La mort dans l'art contemporain », Frontières, vol. 9, $\mathrm{n}^{\mathrm{0}}$ 1, p. 9-19.

GADAMER, H.-G. (1995). «La mort comme question », Langage et vérité, Paris, Gallimard, p. 113-127.

GEORGES, E. (1982). Voyages de la mort, Paris, Berger-Levrault.

HAGNON, M.-E. (2010). «Textures et textiles de corps », Le Devoir, 14 mars 2010, p. E8.

KLIMA, A. (2002). "The charnel ground. Visions of death in Bouddhist ascesis and the redemption of mechanical reproduc- tion ", dans The Funeral Casino, Princeton, Princeton University Press.

MONTPETIT, C. (2010). «La vie avant la mort », Le Devoir, 18 mars 2010.

NEBREDA, D. (2000). Autoportrait, Paris, Léo Scheer, 187 p.

\section{Notes}

1. Premièrement, le moine et l'artiste ont un statut social et professionnel spécial et différent du reste de la population: sur les plans symbolique (figures mythiques imposant le respect) et économique (individus vivant de dons et de subventions), la collectivité leur accorde des avantages. Deuxièmement, ils placent au centre de leur vie leur rapport à l'expérience: qu'il s'agisse d'introspection ou d'extraversion, l'expérience vécue est le terreau de leur réflexion. Troisièmement, ils s'investissent dans leur pratique de manière dévouée et exemplaire: ils choissent délibérément une forme d'isolement et/ou de discipline pour mener à bien leur entreprise. Quatrièmement, ils font les choses souvent de manière plus intense que les «profanes»: notamment en termes de discipline, d'expériences extrêmes, de passion. Cinquièmement, ils jouent un rôle pédagogique en tant que maître, guide ou modèle. Pour toutes ces raisons, ils forment en quelque sorte un moteur de conscientisation de l'expérience dans un premier temps vécue (moine/artiste) et donnée à vivre aux laïcs et aux spectateurs à travers leur témoignage (c.-à-d. enseignements ou œuvres selon). Ces éléments ne sont pas exclusifs à ces pratiques et s'inscrivent parfois dans une vision idéalisée du moine ou une vision du mythe de l'artiste. Par ailleurs, la comparaison est intéressante précisément $\mathrm{du}$ fait que ces pratiques, à première vue si éloignées, se rejoignent néanmoins dans le sens commun.

2. La contemplation de charnier n'est pas exclusive à la méditation bouddhiste theravada, elle est également présente dans de nombreuses traditions du dharma.

3. Le canon pali est constitué de trois principales parties: les règles monastiques, les exposés philosophiques et les discours du Bouddha. C'est à ces derniers, Satipatthanasutta, que Boisvert (1995) se réfère pour son étude. Un siècle plus tard, le Visuddhimagga constitue l'exégèse du canon pali. Boisvert se réfère essentiellement au chapitre 6 de ces écrits postcanoniques (voir Boisvert, 1995, p. 32).

4. La plupart des œuvres citées dans cet article sont accessibles sur Internet. Ainsi, à moins d'indication contraire, une recherche par nom d'artiste et/ou titre de l'œuvre dans un moteur de recherche d'images conduira facilement à la référence citée. Par ailleurs, lorsque l'artiste possède un site Internet officiel, l'adresse est présentée en note.

5. L'image de la performance de Oldenbourg n'est pas disponible en ligne. Une image de celle-ci se trouve par ailleurs en couverture de l'ouvrage de Paul Ardenne, Extrême: esthétiques de la limites dépassée, paru en 2006 chez Flammarion.

6. La démarche personnelle de Nebreda postule la négation de son être. Non sans paradoxe, la pratique artistique de ce dernier documente les manifestations de persécution qu'il inflige à son corps (voir Nebreda, 2000).

7. Le site Internet officiel de Savadov présente plusieurs photographies de cette série. $<$ http://www.savadov.com/>

8. Ce dernier point est paradoxal, puisque dans le culte bouddhiste une grande place est accordée aux ancêtres. L'imaginaire de l'horreur est en grande partie fondée sur ces âmes errantes portant le fardeau de leur histoire singulière, en particulier lorsque la mort s'est produite dans un contexte d'opprobre social.

9. Pour une description de ces pratiques dans des temples au Japon, voir: «Les momies japonaises, des bonzes entrés vivants dans l'immortalité » dans Georges, 1982, p. 251-254.

10. Les charnel grounds sont des forêts-cimetières où sont enterrées des personnes dont la mort est scandaleuse (meurtre, femmes décédées en couches, fœtus, etc.). Ils forment un des lieux les plus tourmentés de l'imaginaire bouddhiste et sont recherchés par certains méditants pour y mener des retraites. L'objectif est d'y exercer la stabilité de l'esprit, bref d'y éprouver sa capacité à rester concentré malgré la mort qui hante ces lieux. Voir Klima, 2002, p. 176-188.

11. Le terme nimitta signifie: une image mentale vivide ou une vision d'une partie du corps, d'un organe ou encore d'un cadavre en décomposition qui surgit durant la méditation sur la répugnance du corps (Akincano, s.d., notre traduction).

12. Cette formule est utilisée par Depraz (1999, p. 26) pour présenter la variation eidétique.

13. Il s'agit ici d'un point de vue occidental. Dans plusieurs traditions du monde oriental certaines morts sont jugées taboues et indignes. L'avorton en fait partie, et en ce sens un autre type de lecture pourrait très bien être proposé. Par ailleurs, les propos de l'artiste Sun Yuan confirment cette idée lorsqu'il dit: «Réfléchir à des problèmes fondamentaux touchant à l'essence de l'homme nous intéresse davantage » (Dawei, 2001, p. 61).

14. L'œuvre de Ducan est décrite par Ardenne dans Extrême: La limite de l'esthétique dépassée, p. 424-425. Par ailleurs, une présentation de l'œuvre par l'artiste est disponible à l'adresse suivante: $<$ http://www.johnduncan. org/blind_date $>$

15. Ardenne rappelle quelques cas médiatisés de nécrophilie; voir "Nécrophilie, crime, mise en forme» et "Macabres trophées (sculpter la viande morte)», dans Ardenne, 2006, p. 416-424.

16. $<$ http://www.damienhirst.com/>; site présentement en construction.

17. <http://www.janasterbak.com/>

18.<http://www.gligorov.aeroplastics.net/>

19. <http://www.cindysherman.com/> 